\section{Envejecimiento y salud: un cambio de paradigma ${ }^{1}$}

1 Basado en: Organización Panamericana de la Salud, División de Promoción y Protección de la Salud. Salud de las personas de edad. Envejecimiento y salud: un cambio de paradigma. Washington, D.C.: OPS; 1998. (Documento inédito CSP25/12).
La salud de las personas de edad es un componente clave para el desarrollo socioeconómico de la Región de las Américas. Entre las razones de esta importancia se puede citar la esperanza de vida, que a principios de los años cincuenta promediaba los 51 años y actualmente supera los 68, llegando incluso a los 75 años en varios países. Este tipo de cambio de la población plantea un reto importante para la salud pública, particularmente en países que todavía no han superado problemas básicos del desarrollo y donde la pobreza persistente genera mayores presiones sobre sistemas que ya están de por sí sobrecargados (1).

La mayoría de los países de la Región atraviesan una etapa intermedia de transición demográfica, en la cual las inversiones dirigidas a la salud de los niños y los adolescentes siguen teniendo una gran prioridad, mientras que las necesidades de salud de las personas de edad y el establecimiento de infraestructuras para una sociedad que va envejeciendo rara vez reciben la atención que deberían. Por otra parte, en los países que se hallan en una etapa más adelantada de la transición demográfica ya se reconoce la necesidad de evaluar los modelos de prestación de servicios de salud a las personas de edad y de lograr que se mantengan los sistemas de pensiones y asistencia sanitaria, pese a las exigencias cada vez mayores que impone el rápido crecimiento del segmento de personas de edad avanzada (75 años o más) en la población.

$\mathrm{Si}$ bien las dificultades que plantean las necesidades sanitarias, sociales y económicas de las personas de edad avanzada varían considerablemente dentro de la Región, hay un principio común para la acción que radica en concentrar esfuerzos para promover la salud y reducir la dependencia de este grupo de población.

\footnotetext{
Alcance del desafío

El envejecimiento de la población repercute sensiblemente sobre varios factores del desarrollo y el funcionamiento de las sociedades, así como sobre el bienestar, no solo de las personas de edad sino de los grupos más jóvenes. De esos factores, los más importantes son los sistemas de pensión y jubilación; la composición y modalidades de participación de la población activa; los arreglos en cuanto a familia y hogar; las transferencias intrafamiliares de
} 
una generación a otra; y las condiciones de salud de las personas de edad (2). La importancia relativa de cada uno de estos aspectos varía, de acuerdo con las particularidades de los regímenes demográficos y las idiosincrasias institucionales de los países. De todas maneras, tarde o temprano ningún país podrá dejar de incluir entre sus asuntos prioritarios de salud pública y economía el tema de las repercusiones del envejecimiento de la población.

Dimensiones demográficas. Las tendencias demográficas que se están produciendo en la Región exigen atención inmediata. Para el año 2000, los Estados Unidos de América y el Canadá cuentan en conjunto con más de 50 millones de personas de 60 años de edad o más, cifra que en América Latina y el Caribe asciende a 42 millones. En 2020, 12,4\% de la población de América Latina y el Caribe (es decir, 82 millones de personas) tendrá 60 años o más (3).

Obviamente, el índice de envejecimiento en los países de la Región no puede tener un comportamiento único y homogéneo. De hecho, habrá una gran heterogeneidad entre países - $y$ aun dentro de cada país- en relación con el momento, la magnitud y las modalidades del proceso de envejecimiento de la población. En general, puede decirse que el momento y la velocidad de las disminuciones de la fecundidad pasadas determinarán el momento y la velocidad del envejecimiento de la población. Asimismo, los cambios futuros en cuanto a mortalidad en la edad adulta y la vejez configurarán la distribución etaria de la población de más edad, sobre todo en cuanto a las proporciones relativas de los estratos de personas más jóvenes (60 a 74 años) y mayores (75 años o más), determinando así una de las características básicas del proceso de envejecimiento. En casi todos los países del mundo, el grupo poblacional que más rápido crece es el de las personas de edad más avanzada. En 1990, en la Región dicho grupo sumaba 21,5 millones de personas, cifra que aumentará más del doble cada 20 años, de modo que para 2020 habrá en total unos 45,9 millones de personas de 75 años de edad o más (4).

Otra forma de observar el aumento en la edad poblacional en la Región consiste en examinar el índice de envejecimiento de determinados países, el cual mide la proporción de personas de 60 años o más por cada 100 menores de 15 años. En el Brasil, dicho índice aumentará de 24 en 1995, a 58 en 2020 y a 74 en 2025; en Chile en los mismos años pasará de 32 a 67 y a 110, y en Cuba de 54 a 107 y a 159. En casi todos los países el índice de envejecimiento se duplicará en los próximos dos decenios, fenómeno que representa un cambio poblacional sin precedentes (4).
Dimensiones epidemiológicas. Con el envejecimiento de la población, la muerte se convierte cada vez más en un fenómeno propio de la vejez. En Argentina, Barbados, Chile, Costa Rica, Cuba, Trinidad y Tabago y Uruguay, más de 55\% del total de defunciones se da entre personas de 65 años o más (2). En 1996, casi 25\% de todas las defunciones en Estados Unidos correspondieron a mujeres mayores de 80 años. Estas cifras indican las tendencias que se observarán en la mayor parte de los países de la Región en los próximos 20 años.

Con el envejecimiento de la población también cambian los tipos de enfermedades predominantes. Las afecciones isquémicas del corazón y las enfermedades cerebrovasculares son las principales causas de defunción en personas de edad, seguidas por las neoplasias y las enfermedades respiratorias, principalmente la neumonía. Conforme aumenta la proporción de personas de edad avanzada, también lo hace el porcentaje de la población que padece enfermedades crónicas y discapacidad, generando una mayor necesidad de recursos sanitarios para estos pacientes, mientras que los costos de la atención de casos agudos o curables permanecen bastante constantes.

Estudios realizados en Canadá, Estados Unidos y el Reino Unido indican que en estos países quienes hoy tienen 65 años o más están menos discapacitados que los miembros de cohortes anteriores a esa misma edad. ${ }^{2}$ Este tipo de investigaciones demuestran que las discapacidades que a menudo se relacionan con el envejecimiento son susceptibles de modificarse y que alcanzar una edad avanzada no es por fuerza sinónimo de discapacidad y enfermedad.

Dimensiones socioeconómicas. Las repercusiones socioeconómicas del envejecimiento poblacional en la Región van a ser mayores principalmente por la velocidad con que ocurre este fenómeno. Cuando la proporción de personas de 15 años o menos en relación con las de 60 o más cae abruptamente, se produce un problema de adaptación por parte de las estructuras sociales y económicas. También en este sentido, la tendencia hacia hogares encabezados por mujeres y el número cada vez mayor de mujeres que participan en el mercado laboral están cambiando la composición y la dinámica de la familia nuclear. Como la atención dispensada por la familia es el elemento básico del cuidado de las personas de edad cuya salud es frágil, los gobiernos se verán obligados a formular programas de respaldo a las familias en su función de prestadoras de cui-

\footnotetext{
2 Denver Summit of the Eight (G8 countries). Active aging: A shift in the paradigm. 1997. (Documento inédito).
} 
dados. En Japón, por ejemplo, el envejecimiento rápido de la población provocó un aumento en el número de personas obligadas a renunciar al empleo para hacerse cargo de sus responsabilidades en la familia. En Estados Unidos, las inversiones en asistencia sanitaria domiciliaria aumentaron de US\$ 20000 millones en 1980 a $\$ 64000$ millones en 1990 y $\$ 98500$ millones en 1994 (5).

El incremento en el número y la proporción de personas de edad en la Región repercutirán sensiblemente sobre los gastos en atención de salud, las clases de instituciones y arreglos informales que se requerirán en apoyo del cuidado familiar, y la distribución de recursos a lo largo de la vida.

\section{La equidad y la salud de las personas de edad}

Las cuestiones de salud relacionadas con el crecimiento del grupo poblacional de más edad abarcan aspectos de equidad importantes. Ante todo, la salud en la vejez depende mucho de los modos de vida, la exposición a factores de riesgo y las oportunidades de acceso a la protección y la promoción de la salud. Sin embargo, la posibilidad de contar con una atención sanitaria de buena calidad varía mucho entre los distintos estratos socioeconómicos. Sin estrategias nacionales para tratar cada uno de estos factores con justicia, las desigualdades de calidad de vida y bienestar de las personas de edad de diferentes clases socioeconómicas no harán sino ahondarse.

Si bien en la Región la esperanza de vida al nacer varía enormemente según el grado de desarrollo de los países, la esperanza de vida a los 60 años es relativamente uniforme. Una persona pobre que llega a cumplir esta edad tiene una esperanza de vivir aproximadamente 20 años más, independientemente de dónde habite. Sin embargo, se sospecha que para la mayoría de los pobres la ganancia en esperanza de vida se ve contrarrestada en gran medida por la mayor discapacidad causada por una o varias enfermedades crónicas (1).

Asimismo hay que considerar las diferencias derivadas del sexo, porque el hombre y la mujer están expuestos a modalidades de mortalidad diferentes y se ven afectados por problemas de salud muy distintos. La mujer, que tradicionalmente ha participado mucho menos en la fuerza laboral, tiene un acceso limitado a la obtención de ingresos y servicios esenciales. En la vejez las mujeres, sobre todo las viudas sin apoyo familiar, son quienes corren el mayor riesgo de sufrir un deterioro en su calidad de vida (2). Por otra parte, los hombres generalmente mueren a una edad más temprana que las mujeres.

El crecimiento del número de personas de edad avanzada se verá acompañado por diferencias significativas entre cohortes. En la medida en que la participación en la fuerza laboral y los logros educacionales influyen en el nivel de activos e ingresos de una persona, las cohortes de menos y de más edad experimentarán grandes diferencias en lo referente a sus posibilidades de acceso a los recursos esenciales para una vida sana. Cabe entonces a los responsables de formular y ejecutar políticas la tarea de procurar que la población de edad avanzada tenga como mínimo niveles de bienestar aceptables, para lo cual un requisito es reducir las diferencias en las condiciones de salud de las personas a cualquier edad.

\section{La labor de la OPS en el área del envejecimiento}

Marco histórico. En 1980, el 27. ${ }^{\circ}$ Consejo Directivo aprobó la resolución CD27.R16, en la que manifestaba preocupación por la falta de programas adecuados para las personas de edad e instaba a los Estados Miembros a establecer programas eficaces. En junio de 1981, el Comité Ejecutivo de la OPS, tras haber analizado en su 86. ${ }^{\mathrm{a}}$ sesión el tema de la asistencia sanitaria a las personas de edad, aprobó la resolución CE86.R30, en la que se recomendaba a los Estados Miembros promover la salud y el bienestar de las personas de edad, elaborar programas integrales para satisfacer sus necesidades sanitarias, integrar los programas de asistencia sanitaria en la estrategia de atención primaria y tener en cuenta plenamente los factores socioeconómicos pertinentes.

En agosto de 1982, en Viena, la Asamblea Mundial de las Naciones Unidas sobre el Envejecimiento aprobó el Plan de Acción Internacional sobre Envejecimiento. Por su parte, en 1985 el $37 .^{\circ}$ Consejo Directivo de la OPS aprobó la elaboración de un programa sobre la Salud del Adulto y las Personas de Edad, donde se exhortaba a los Estados Miembros a seguir esforzándose por conseguir recursos extrapresupuestarios para el Programa de Salud de las Personas de Edad.

Resultados logrados hasta la fecha. Un examen de las políticas, planes y programas nacionales en favor de las personas de edad permitió constatar que la actividad legislativa ha estado relacionada principalmente con políticas y medidas de jubilación y pensión dirigidas a proteger a la población frente a la pobreza extrema y con la ampliación del acceso a la atención de salud. En 1996, el Parlamento Latinoamericano, en colaboración con la OPS, elaboró una ley modelo para el cuidado integral de las personas de edad.

En julio de 1997 se realizó en Montevideo un foro de políticas públicas sobre el envejecimiento 
poblacional, que contó con respaldo político y gubernamental de primer nivel. En esta reunión, 115 delegados de 21 países de la Región redactaron la Declaración de Montevideo sobre Políticas Integrales para el Envejecimiento Sano. De manera análoga, en mayo de 1998 se llevó a cabo en Nassau el Foro del Caribe sobre Salud y Envejecimiento, que emitió la Carta del Caribe sobre Salud y Envejecimiento.

Recursos humanos. Una barrera grave que se presenta a la hora de formular planes y programas nacionales de salud en favor de personas de edad avanzada radica en la falta de educación gerontológica y geriátrica que tienen los profesionales de la atención primaria, y los pocos conocimientos en gestión de programas con que cuentan los gerontólogos y geriatras. Desde 1990 la OPS viene haciendo hincapié en el desarrollo de aptitudes de liderazgo en enfermería gerontológica. En tal sentido ha realizado un estudio sobre el adiestramiento gerontológico en las facultades de odontología y ha promovido una encuesta regional sobre la enseñanza de la gerontología y la geriatría.

En 1998 el Programa de Salud de la Familia y Población, en colaboración con el Programa de Coordinación de Investigaciones, llevó a cabo un estudio multicéntrico sobre la salud y el bienestar de las personas de edad. El estudio tiene por objeto recopilar información sobre el estado y los problemas de salud de las personas de edad en siete países de América Latina y el Caribe que representan una amplia gama de situaciones demográficas y contextos institucionales, así como evaluar y analizar las diferencias de cohorte, sexo y estado socioeconómico con respecto a la situación sanitaria, el acceso a los servicios de salud y su aprovechamiento. Los países seleccionados para el estudio fueron Barbados, Brasil, Chile, Costa Rica, Cuba, México y Uruguay.

Por otra parte, en colaboración con el Centro Latinoamericano y del Caribe para Información en Ciencias de la Salud (BIREME), institución de la OPS en São Paulo, se ha creado una base de datos sobre envejecimiento y salud a la que se tiene acceso por Internet.

Direcciones futuras. El marco conceptual de la OPS se basa en la premisa de que el buen envejecimiento depende en gran medida de la prevención de las enfermedades y la discapacidad, del mantenimiento de una gran actividad física y de las funciones cognoscitivas, y de la participación ininterrumpida en actividades sociales y productivas.

Sobre la base de las enseñanzas aprendidas de la comunidad internacional, y según las perspectivas adoptadas por el Programa Mundial de la OMS sobre el Envejecimiento, el plan de acción de la OPS presenta un método holístico relativo a la salud y el bienestar de las personas de edad, que incorpora las perspectivas siguientes: a) del ciclo vital en su totalidad; b) de promoción de la salud; c) de género; d) de carácter intergeneracional, y e) de carácter ético. A continuación se reseñan los elementos fundamentales de dicho plan.

- El envejecimiento es un proceso que dura toda la vida, dado que los modos de vida que favorecen un envejecimiento sano se forman a temprana edad. El buen envejecimiento o envejecimiento sano no depende únicamente de la ausencia de enfermedades, sino también de la ausencia, presencia o gravedad de factores de riesgo de enfermedad. La unidad de Envejecimiento y Salud, en colaboración con el Programa de Alimentación y Nutrición de la División de Promoción y Protección de la Salud, el Programa de Enfermedades No Transmisibles de la División de Prevención y Control de Enfermedades y el Programa Especial de Vacunas e Inmunización, actualmente preparan programas de promoción de la salud y prevención de las enfermedades centrados en el ejercicio físico, la nutrición y la vacunación de las personas de 60 años de edad o mayores.

- Muchas personas de edad pueden llegar a estar jubiladas durante 20 años o más. El tener dinero suficiente para mantenerse se convierte en uno de los problemas más acuciantes para estas personas, sobre todo las que tienen escasa educación formal y se enfrentan con problemas de salud. El buen envejecimiento requiere hacer hincapié en la educación a lo largo de toda la vida, el uso creativo de las experiencias vitales y las políticas que alienten la utilización de trabajadores de edad. Se necesitan programas de preparación para la jubilación, al igual que políticas nacionales que garanticen un nivel mínimo y adecuado de seguridad financiera para las personas de edad jubiladas o desocupadas.

- El aislamiento físico y emocional es un factor de alto riesgo que afecta adversamente a la salud y al bienestar de las personas de edad, mientras que el apoyo social, tanto emocional como material, puede tener efectos favorables en la salud. A fin de fomentar la cohesión y fortalecer la interdependencia de las generaciones, el programa de la OPS sobre Envejecimiento y Salud colaborará con las organizaciones no gubernamentales (ONG) y los Estados Miembros para establecer programas y actividades eficaces que reduzcan el aislamiento generacional.

- La adaptabilidad a lo largo de la vida es un elemento esencial para un buen envejecimiento (6). Dicho concepto se relaciona con la capacidad de 
una persona para recuperarse de las pérdidas, los acontecimientos estresantes y las enfermedades que a menudo acompañan al proceso de envejecimiento. Conforme las investigaciones en este ámbito vayan arrojando nueva luz sobre los factores determinantes del buen envejecimiento, la unidad de Envejecimiento y Salud del Programa de Salud de la Familia y Población, con la colaboración del Programa de Modos de Vida Sanos y Salud Mental, identificará estrategias eficaces para tratar la salud mental de las personas de edad y servirá como centro de intercambio de información.

- Las cuestiones éticas propias de una sociedad que envejece están presentes a todo nivel en las políticas públicas. El programa de la OPS centrará casi toda su atención en dos asuntos fundamentales. El primero de ellos, relacionado con la ética clínica, gira en torno a la toma de decisiones informada, sobre todo en lo que respecta a las decisiones sobre calidad de vida y las opciones en cuanto a intervención médica en la atención de los moribundos; el segundo, en conexión con la ética social, se relaciona con el respeto y la justicia entre las generaciones. El envejecimiento poblacional, junto con los adelantos de la tecnología médica y el movimiento hacia la privatización y la descentralización tanto de los recursos como de la toma de decisiones, permite tener la certeza de que el tema de la equidad intergeneracional será objeto de debates públicos frecuentes.

Estrategias regionales de la OPS para lograr el envejecimiento sano y el desarrollo. El plan de acción de la OPS para 1999-2002 sigue el marco conceptual enunciado en la sección anterior. La meta: promover el buen envejecimiento. El plan de acción de la OPS procura promover la salud y el bienestar de las personas de edad mediante la creación y el fortalecimiento de programas y servicios nacionales y locales. También se propone impulsar iniciativas para idear intervenciones de promoción de la salud y prevención de las enfermedades dirigidas a las personas de edad; asignar funciones creativas para estas personas en la sociedad, y crear entornos propicios para las personas de edad discapacitadas y sus familias.

Componentes programáticos clave. Si bien las actividades del plan de acción tienen por objeto beneficiar en definitiva a las personas de edad de la Región, su foco de atención es el desarrollo de la infraestructura y las posibilidades que se requieren para satisfacer las necesidades de dichas personas y sus familias. Ello incluye el establecimiento de apti- tudes organizativas y el desarrollo de recursos humanos con el adiestramiento, las herramientas y las oportunidades para obrar con eficacia en el seno de esas estructuras y programas. En el cuadro 1 se presentan las estrategias para poner en ejecución los componentes pragmáticos clave de la OPS.

Las instituciones e instrumentos fundamentales para dar comienzo a las actividades en los países y generar inversiones a nivel nacional en el ámbito de los recursos humanos y financieros son los ministerios de salud y los programas nacionales sobre envejecimiento. Hacen falta socios que asuman un compromiso en pro del plan de acción mediante la participación en proyectos de colaboración, subsidios para la investigación, grupos consultivos de expertos, programas de adiestramiento y talleres. Entre los socios potenciales cabe mencionar a los ministerios de trabajo, bienestar social, justicia, educación y seguridad social; los gobiernos locales (municipales); las ONG que trabajan directamente con personas de edad; las universidades y los institutos de investigación; las organizaciones de jubilados; los medios de comunicación; las aseguradoras, y otras organizaciones del sector privado que trabajan con personas de edad. El fomento de las relaciones entre los países de la Región por medio de la colaboración horizontal se seguirá utilizando como estrategia operativa de gran eficacia. La colaboración con otros programas y divisiones de la OPS será un factor esencial para la ejecución del plan de acción, por lo que se la seguirá fomentando.

\section{Plan de acción 1999-2002}

Fortalecimiento de las bases de información e investigación. La OPS procurará proporcionar a los Estados Miembros información y datos confiables sobre cuestiones relacionadas con el envejecimiento, incluido el emprendimiento de iniciativas de investigación en toda la Región. Las tareas de investigación patrocinadas por la OPS estarán orientadas por las siguientes preguntas:

- ¿Cuán sano es en la Región el grupo de 60 años o más de edad? ¿Qué clases de dolencias padecen sus miembros, y cómo varía este factor según el nivel de ingresos, el sexo y las categorías educacionales, y según se trate de un entorno rural o urbano? ¿Cuáles son las probabilidades de que el estado de salud de las personas de edad cambie conforme crecen los niveles de ingresos y de educación?

- ¿Cuáles son los factores determinantes del envejecimiento sano? ¿Qué intervenciones en cuanto a promoción de la salud contribuyen a que las personas envejezcan manteniéndose activas? 
CUADRO 1. Estrategias para los componentes programáticos claves de la OPS

\begin{tabular}{|c|c|c|}
\hline $\begin{array}{l}\text { Componentes } \\
\text { programáticos }\end{array}$ & $\begin{array}{c}\text { La salud de las personas de edad } \\
\text { Se concentra en los servicios } \\
\text { de salud, las cuestiones asistenciales } \\
\text { y las opciones de la comunidad frente a } \\
\text { la necesidad de confinamiento en instituciones }\end{array}$ & $\begin{array}{c}\text { Las personas de edad como agentes de cambio } \\
\text { Se concentra en redes de personas } \\
\text { jubiladas y programas intergeneracionales }\end{array}$ \\
\hline Investigación & $\begin{array}{l}\text { Estudio multicéntrico sobre la situación de salud de } \\
\text { las personas de edad, principalmente epidemiológico } \\
\text { y longitudinal. Proyectos conjuntos con universidades } \\
\text { y centros colaboradores. }\end{array}$ & $\begin{array}{l}\text { Investigación aplicada con centros colaboradores y } \\
\text { universidades acerca de buenas prácticas para pro- } \\
\text { mover la actividad física, la evaluación nutricional y la } \\
\text { integración social de las personas de edad. Grupos de } \\
\text { expertos formados por personas jubiladas. }\end{array}$ \\
\hline $\begin{array}{l}\text { Difusión de } \\
\text { información }\end{array}$ & $\begin{array}{l}\text { Normas, manuales, instrumentos y metodología para } \\
\text { la investigación sobre el envejecimiento, documentos } \\
\text { de posición, mensajes por distintos medios. }\end{array}$ & $\begin{array}{l}\text { Normas y manuales de adiestramiento para la elabo- } \\
\text { ración de programas comunitarios sociales y terapéu- } \\
\text { ticos para las personas de edad, capacitación de los } \\
\text { prestadores de asistencia, "bancos de talento", progra- } \\
\text { mas intergeneracionales, programas de carácter vo- } \\
\text { luntario y generadores de ingresos. }\end{array}$ \\
\hline $\begin{array}{l}\text { Promoción de } \\
\text { la causa }\end{array}$ & $\begin{array}{l}\text { Redes multisectoriales; uso de los medios de comuni- } \\
\text { cación y adiestramiento como promotores de la causa } \\
\text { de profesionales de la salud jubilados. }\end{array}$ & $\begin{array}{l}\text { Materiales educativos para el Día Mundial de la Salud } \\
\text { y el Año Internacional de las Personas de Edad. Red } \\
\text { de universidades de la tercera edad. }\end{array}$ \\
\hline $\begin{array}{l}\text { Desarrollo de } \\
\text { recursos humanos }\end{array}$ & $\begin{array}{l}\text { Normas para elaborar programas de estudios de } \\
\text { gerontología, instrumentos clínicos para trabajadores } \\
\text { de atención primaria de salud. Cursos multiplicadores, } \\
\text { educación a distancia, red de centros de excelencia. }\end{array}$ & $\begin{array}{l}\text { Materiales didácticos para adiestrar como promotores } \\
\text { de salud a personas de edad. }\end{array}$ \\
\hline $\begin{array}{l}\text { Políticas, planes } \\
\text { y programas }\end{array}$ & $\begin{array}{l}\text { Programas nacionales y colaboración multisectorial. } \\
\text { Normas y manuales de adiestramiento para elabora- } \\
\text { ción de políticas y programas. Instrumentos para eva- } \\
\text { luar servicios de atención primaria de salud, progra- } \\
\text { mas comunitarios y asistencia prolongada en diversos } \\
\text { entornos. }\end{array}$ & $\begin{array}{l}\text { Normas para proyectos gerontológicos en municipios } \\
\text { saludables e indicadores de entornos propicios para } \\
\text { las personas de edad y sus familias. }\end{array}$ \\
\hline $\begin{array}{l}\text { Movilización } \\
\text { de recursos }\end{array}$ & $\begin{array}{l}\text { Propuestas para conseguir subvenciones. Colabora- } \\
\text { ción intersectorial e interinstitucional y trabajo con } \\
\text { centros colaboradores regionales. }\end{array}$ & $\begin{array}{l}\text { Trabajo con organizaciones de jubilados, programas } \\
\text { nacionales de seguro social y ministerios de salud. }\end{array}$ \\
\hline $\begin{array}{l}\text { Colaboración } \\
\text { técnica directa }\end{array}$ & $\begin{array}{l}\text { Creación de redes de consultores nacionales y } \\
\text { regionales. }\end{array}$ & $\begin{array}{l}\text { Establecimiento de redes con consultores nacionales } \\
\text { y regionales. }\end{array}$ \\
\hline
\end{tabular}

- Dadas las necesidades y los valores cambiantes de la familia, ¿qué clases de programas comunitarios son los de mayor eficacia a la hora de evitar el internamiento innecesario en establecimientos asistenciales y fomentar una vida independiente y digna en la tercera edad?

- ¿Qué establecimientos y servicios son usados por las personas de edad y cómo varían según el nivel de ingresos, el sexo y la educación de los consumidores, el precio y la disponibilidad de los establecimientos y la ubicación en una zona rural o urbana?

- ¿Cómo afecta la conducta de los sistemas de seguro de salud al envejecimiento de la población? ¿Qué problemas especiales se les plantean a las personas de edad más pobres? ¿Qué mecanismos de financiación permitirán evitar estos problemas en los planes públicos y privados?

El temario de investigación de la OPS debe formularse en cooperación con centros colaboradores de la OPS/OMS, como el Instituto Nacional del
Envejecimiento de los Estados Unidos, universidades e institutos de investigación, instituciones financieras internacionales como el Banco Mundial y el Banco Interamericano de Desarrollo, y otros organismos del sistema de las Naciones Unidas.

Difusión de información. Una función importante del programa de la OPS sobre envejecimiento y salud es fortalecer las redes de la Región para poder hacer de centro de distribución de información sobre envejecimiento y salud. La página inicial de la OPS sobre envejecimiento y salud en la World Wide Web no solo servirá como depósito de información, sino que además hará encuestas y recibirá datos sobre distintas cuestiones y tendencias relacionadas con la formulación de planes, programas y servicios para el envejecimiento. Sin embargo, la OPS es perfectamente consciente de que la mayor disponibilidad de información por la Internet muy bien podría ensanchar la brecha entre quienes tie- 
nen acceso a la tecnología y quienes no. Por consiguiente, el uso de herramientas más tradicionales como material impreso, audiocintas y videocintas seguirá siendo un medio importante para la difusión de información.

\section{Desarrollo de la comunicación social y promoción de la causa}

La comunicación social y los medios de comunicación son herramientas poderosas para promover cambios. Por lo tanto, si se quiere inculcar a lo largo de la vida el nuevo paradigma de envejecer manteniéndose activo, los comunicadores y los educadores de salud de la Región deben comprenderlo. Los medios de comunicación pueden ayudar a cambiar las imágenes del envejecimiento y a crear una cultura de solidaridad intergeneracional en respaldo de los cambios demográficos que ocurren en la Región. La OPS propone elaborar videocintas, anuncios de servicio público y estrategias de comunicación para difundir mensajes sobre la forma de envejecer con salud y manteniéndose activo, y también propone talleres regionales para los educadores sanitarios y los medios de comunicación, destinados a reforzar su capacidad para transmitir mensajes de promoción del envejecimiento sano.

Desarrollo de recursos humanos. La necesidad de invertir en capital humano para una sociedad que envejece tiene carácter crítico. El programa de estudios y de adiestramiento para los profesionales de la atención primaria de salud ha hecho hincapié en la asistencia de los niños y las madres. Por el contrario, en prácticamente ninguno de los inventarios de materiales didácticos y programas de enseñanza permanente existe información sobre cómo identificar y tratar los problemas de salud relacionados con el envejecimiento. La Organización establecerá una red de centros de excelencia sobre envejecimiento y salud en la Región, que se encargarán de: a) formular directrices para la enseñanza multidisciplinaria de la gerontología y la geriatría a nivel tanto de pregrado como de posgrado; b) elaborar guías prácticas, módulos didácticos y otros recursos educacionales dirigidos a los trabajadores de atención primaria de salud; c) promover la formulación de directrices para la enseñanza del envejecimiento sano en los programas de estudios escolares relativos a la salud, y d) fomentar el aprendizaje a lo largo de la vida entre las personas de edad.

Se establecerán asociaciones con fundaciones privadas a fin de preparar una iniciativa regional para el desarrollo de los cuerpos docentes y la realización de programas de adiestramiento para edu- cadores, en los que se utilizará una combinación de tecnologías para el aprendizaje a distancia con reuniones intensivas de corta duración.

Formulación de políticas, planes y programas. Conforme aumente la demanda de colaboración técnica regional, la OPS abordará esa necesidad fortaleciendo la capacidad de una red ad hoc regional de asesores sobre políticas públicas y desplegando actividades continuas de adiestramiento.

La formulación de programas y servicios debe concentrarse en tres ámbitos específicos: a) programas comunitarios integrales que proporcionan toda una gama de entornos para el envejecimiento sano, y programas concebidos para apoyar las actividades de cuidado familiar, la protección de la dignidad de las personas de edad y la prevención del internamiento innecesario en establecimientos asistenciales de las personas de edad cuya salud es frágil; b) programas formulados para fortalecer la capacidad del nivel de atención primaria de salud en lo que hace al mejoramiento de la calidad de la atención proporcionada a las personas de edad, contribuyendo así a evitar la utilización de las salas de urgencias de los hospitales públicos, que es más costosa, y c) programas concebidos para ofrecer incentivos capaces de alentar la autonomía, las actividades socialmente productivas y los programas generadores de ingresos para las personas de edad.

Movilización de recursos. Para ejecutar el plan de acción, el programa de la OPS sobre envejecimiento y salud necesita establecer vínculos con distintos programas de la Organización; lograr la movilización de recursos regionales, nacionales, internacionales y privados para complementar los fondos básicos asignados al programa; y crear una red de centros colaboradores especializados en salud y envejecimiento. Los Estados Miembros por su parte deben movilizar recursos suficientes para ejecutar las políticas nacionales, planificar y establecer una infraestructura de servicios de salud y sociales y preparar la fuerza laboral necesaria para hacer frente al envejecimiento poblacional.

La OPS espera movilizar recursos provenientes de instituciones externas para trabajar en distintos ámbitos básicos del envejecimiento y la salud. Con tal propósito, la Organización prevé realizar las siguientes actividades: establecer asociaciones con fundaciones privadas como Novartis y Kellogg para el desarrollo de recursos humanos; colaborar con el Banco Mundial y el Banco Interamericano de Desarrollo para proyectos de investigación y demostración en lo referente al establecimiento de la infraestructura sanitaria y social para los grupos 
poblacionales que van envejeciendo, en el contexto del desarrollo sostenible; y cooperar con universidades, centros colaboradores e institutos de investigación de toda la Región para movilizar cooperación técnica.

\section{SYNOPSIS}

\section{Aging and health: a shift in the paradigm}

The aging of the population due to increasing life spans requires a reevaluation of the health care models that prevail in the countries of the Region of the Americas, given that the illnesses typical of old age make up an ever-increasing share of the morbidity burden. The countries in the Region are in an intermediate stage of demographic transition. They face the challenge of balancing priority care for the health problems characteristic of developing countries along with care for the chronic and debilitating diseases that afflict the elderly. An even greater challenge is gauging, in a timely manner, the health, social, and economic consequences of the demographic transition over the next millennium, to allow the countries to respond to these consequences through an in-depth restructuring of their health and social services.

The extension of human life imposes a new vision of the health of the elderly that is oriented toward self-sufficiency, economic independence, and living life to its fullest. It will not be easy to integrate this new paradigm into the countries' health services, which are now primarily oriented toward curative care and are structured to fulfill the requirements of an epidemiological scenario that is undergoing a rapid transformation. The challenge is even greater because of the wide range of needs that the elderly have across countries with varying degrees of development. Nevertheless, sooner or later the governments of all the countries of the Region will be forced to adopt concrete measures to deal with the problems posed by the population's gradual aging.

\section{REFERENCIAS}

1. Martin LG, Kinsella K. Research on the demography of aging in developing countries. En: Martin LG, Preston SH, eds. Demography of aging. Washington, D.C.: National Academy Press; 1994.

2. Kalache A, Coombes Y. Population aging and care of the elderly in Latin America and the Caribbean. Rev Clin Gerontol 1995;5:347-355.

3. Organización Panamericana de la Salud. Las condiciones de salud en las Américas.
Vol. 1. Washington, D.C.: OPS; 1990. (Publicación Científica No. 524).

4. United Nations. World population prospects. New York: UN; 1994.

5. The Institute for Health and Aging. Chronic care in America: a $21^{\text {st }}$ century challenge. San Francisco: University of California; 1996.

6. Rowe JW, Kahn RL. Successful aging. Gerontol 1997;37:4:433-440.
7. Elo IT, Preston SH. Effects of early-life conditions on adult mortality: a review. Population Index 1992:58(2).

8. Kalache A. ¿Qué repercusiones tiene el envejecimiento de la población en el futuro previsible? En: Pérez EA, ed. La atención de los ancianos: un desafío para los años noventa. Organización Panamericana de la Salud; 1994. (Publicación Científica No. 546). 